\title{
Análise e simulação de antena de microfita para controle e monitoramento de um drone em áreas agrícolas
}

\author{
Leonardo Marques de Freitas ${ }^{[1]}$, Édson Fernandes Chaves Filho ${ }^{[2]}$, Humberto Dionísio de Andrade ${ }^{[3]}$, \\ Matheus Emanuel Tavares Sousa ${ }^{[4]}$ e José Lucas da Silva Paiva ${ }^{[5]}$ \\ [1] Universidade Federal Rural do Semi-Árido; leonardo.xoop@gmail.com \\ ${ }^{[2]}$ Universidade Federal Rural do Semi-Árido; edson.fernandes.chaves.381@gmail.com \\ [3] Universidade Federal Rural do Semi-Árido; humbertodionisio@ufersa.edu.br \\ ${ }^{[4]}$ Universidade Federal Rural do Semi-Árido; matheus.sousa@ufersa.edu.br \\ ${ }^{[5]}$ Universidade Federal Rural do Semi-Árido; lucas.paiva@ufersa.edu.br
}

Recebido: 07/07/2020;

Aceito: 27/07/2020;

Publicado: 11/09/2020.

Resumo: Este artigo tem por objetivo projetar uma antena de microfita para transmissão e recepção de dados de controle de um VANT (Veículo aéreo não tripulado) para o monitoramento de áreas agrícolas. Com o intuito de utilizar as características da antena de microfita para direcionar o sinal de radio frequência para uma direção específica, para melhorar o alcance e qualidade do sinal. Deste modo, a partir de simulações nos softwares Ansys HFSS 2019, Scilab e Mission Planner, demonstrar a viabilidade deste recurso para monitoramento de áreas agrícolas, das quais proporcionam mapas de produtividade, de infestação de pragas e ervas daninha, valores fundamentais para a melhoria de produtividade, sendo possível reduzir um dos problemas que os grandes campos agrícolas possuem, devido a sua extensão, permitindo o VANT a alcançar áreas maiores. A antena de microfita desenvolvida possui sua frequência de ressonância em $2,45 \mathrm{GHz}$ (Banda ISM - Industrial, Cientific and Medical band), além de possuir características diretivas satisfatórias para as aplicações específicas de agricultura de precisão simuladas.

Palavras-chave: VANT; Agricultura de precisão; Antena de microfita.

Abstract: This article aims to design a microstrip antenna for transmitting and receiving control data from an UAV (unmanned aerial vehicle) for monitoring agricultural areas. In order to use the characteristics of the microstrip antenna to direct the radio frequency signal in a specific direction, to improve the range and quality of the signal. Thus, from simulations in the Ansys HFSS 2019, Scilab and Mission Planner software, demonstrate the viability of this resource for monitoring agricultural areas, which provide maps of productivity, pest and weed infestation, fundamental values for improving productivity, being possible to reduce one of the problems that large agricultural fields have, due to its extension, allowing UAV to reach larger areas. The developed microstrip antenna has a resonance frequency of $2.45 \mathrm{GHz}$ (ISM - Industrial, Scientific and Medical band), in addition to having satisfactory directives for specific simulated precision agriculture applications.

Key-words: UAV; Precision agriculture; Microstrip antena.

\section{INTRODUÇÃO}

$\mathrm{D}$ entre as mais diversas tecnologias inovadoras utilizadas para as áreas agrícolas, a inserção de um dispositivo móvel de monitoramento e irrigação foi um grande marco para que a agricultura se modernizasse, tomando proporções que levaram aos diversos estudos da chamada hoje de "agricultura de precisão". Este dispositivo móvel é mundialmente conhecido como "drone", termo utilizado de maneira popular, contudo, quando utilizados de maneira industrial passam a ser conhecidos como VANT’s (Veículos 
Aéreos não tripulados), dos quais não somente foram inseridos na agricultura, mas também em todo em serviços que necessitam principalmente de monitoramento, seja relacionada a segurança ou de uma matéria prima. A principal importância da utilização deste tipo de equipamento é referente a otimização de processos, redução de custos, economia de tempo e maior segurança [1].

Os VANT's são controlados por sinais de radio frequência, no qual possuem normalmente o seu controle de movimento em 2,45 GHz, e aquisição de imagens de voo em 5,85 GHz. Desta maneira, para que se possa transmitir e receber as informações do drone é necessário possuir uma antena que tenha sua frequência de ressonância de acordo. Para isto, tem-se diversos tipos de antenas no mercado, a mais comum de todas é a antena de dipolo, no qual, possui características omnidirecionais, ou seja, tem a capacidade de alcançar todas as direções com a mesma potência e ganho. Contudo, para alguns tipos de áreas a principal importância é alcançar uma maior distância horizontalmente e em somente uma direção, para estes casos, é necessário a antena de microfita simples, que é uma fita metálica de pequena espessura posicionada a uma distância específica de um plano de terra, quando este material delimitador possui características dielétricas específicas, conhecido como substrato [2].

Antenas de microfita possuem diversos tipos de configurações de patch e de alimentação, o tipo mais comum é o tipo de patch retangular e alimentação por linha de transmissão com inset feed. $\mathrm{O}$ termo inset feed é designado a um método de casamento de impedância, que consiste em um par de fendas paralelas a linha de transmissão [3]. As antenas de microfita são utilizados em diversos seguimentos de pesquisa por serem de baixo peso, fácil fabricação e pela adaptabilidade a superfícies planas, se tornando assim bastante atrativas para o uso nos VANT's [4], além disto, possuem baixa radiação de polarização cruzada, são moldáveis e versáteis em relação a frequência de ressonância, polarização, diagrama de radiação e de impedância [2]. Neste trabalho será desenvolvida uma antena de microfita com patch retangular e alimentação por linha de transmissão com inset feed, utilizando-se da faixa ISM com frequência em $2,45 \mathrm{GHz}$ para transmissão e recepção de dados e controle de voo.

\section{MATERIAIS E MÉTODOS}

A antena de microfita de patch retangular foi projetada para operar na faixa de frequência de controle de voo de um VANT $(2,45 \mathrm{GHz})$, foram utilizados os softwares Scilab® e Ansys HFSS 2019®, que possibilitam a simulação da antena para a obtenção de valores referentes a perda de retorno, diagramas de radiação, dentre outros, a partir do modelo da cavidade, linha de transmissão e método do inset feed.

\subsection{Características da antena}

A partir da determinação dos parâmetros iniciais, pôde-se iniciar os cálculos dos parâmetros físicos da antena, no qual, tem-se a necessidade de especificar o substrato, que são caracterizados principalmente pela constante dielétrica, que representa a razão entre a permeabilidade elétrica do material com o vácuo. Dentre os variados materiais utilizados para estudo, o principal é o FR4 (fibra de vidro), que tem sua constante dielétrica igual a 4,4 (Êr), por ser um material de fácil obtenção e de baixo custo. Além disto, define-se a espessura do substrato utilizado, neste quesito, foi utilizado uma placa de FR4 laminada de cobre com espessura de 1,55 mm. Por conseguinte, como o método de alimentação utilizado é através de uma linha de transmissão, definindo a impedância do conector de entrada $(50 \mathrm{Ohm})$, para que desta maneira, através dos modelos prescritos por Balanis [2] e Huang e Boyle [5], adquire-se os valores físicos da antena de acordo com a Figura 1 (a) e (b) e Tabela 1, além de projetar a antena no software Ansys HFSS 2019®, como demonstrado na Figura 1 (c).

TABELA 1. Características físicas da antena de microfita.

\begin{tabular}{c|c|c|c}
\hline Parâmetro & Valor $(\mathbf{m m})$ & Parâmetro & Valor (mm) \\
\hline Largura do patch (W) & 36,30 & Comprimento da linha de transmissão & 14,95 \\
& & $(\mathrm{~lm})$ & 2,29 \\
\hline Comprimento do patch (L) & 28,12 & Largura da linha de transmissão (Wo) & 65,78 \\
\hline Largura do inset feed (yw) & 0,21 & Largura do substrato (Ws) & 57,60 \\
\hline Comprimento do inset feed (yo) & 8,64 & Comprimento do substrato (Ls) & \\
\hline
\end{tabular}




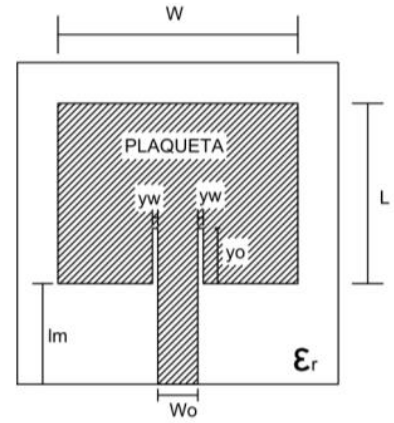

(a)

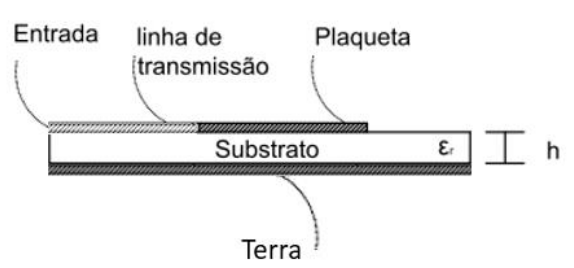

(b)

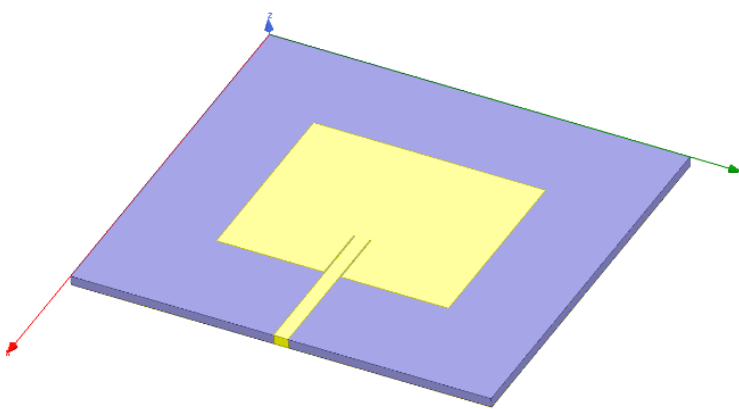

(c)

FIGURA 1. Representação da antena de microfita (a) vista frontal; (b) vista lateral; (c) simulada em software.

\subsection{Simulação referência para antena projetada}

Para a representação de modelos de situações no qual a antena projetada poderia ser utilizada, foi utilizado o software Mission Planner ${ }^{\circledR}$, um mecanismo de controle, programação e simulação de VANTS's em geral, para que se tenha um voo exemplar para análise da antena projetada. Deste modo, foi utilizado uma parte final do campus leste da Universidade Federal Rural do Semi-Árido (UFERSA), representando uma área agrícola simples de aproximadamente 8,3 hectares, como pode-se observar na Figura 2. O centro de controle está localizado em uma das extremidades, como o esperado, pelo fato da antena ser direcional, além disto, é possível observar o drone percorrendo todo o percurso em amarelo, de maneira autônoma, com algumas imagens capturadas em pontos específicos para a obtenção das imagens para análise. Além disto, o VANT utilizado como modelo para as simulações de voo e de modelagem é do tipo F450 (Figura 3), no qual, possui antena do tipo dipolo com frequência de ressonância de acordo com o projetado $(2,45 \mathrm{GHz})$.

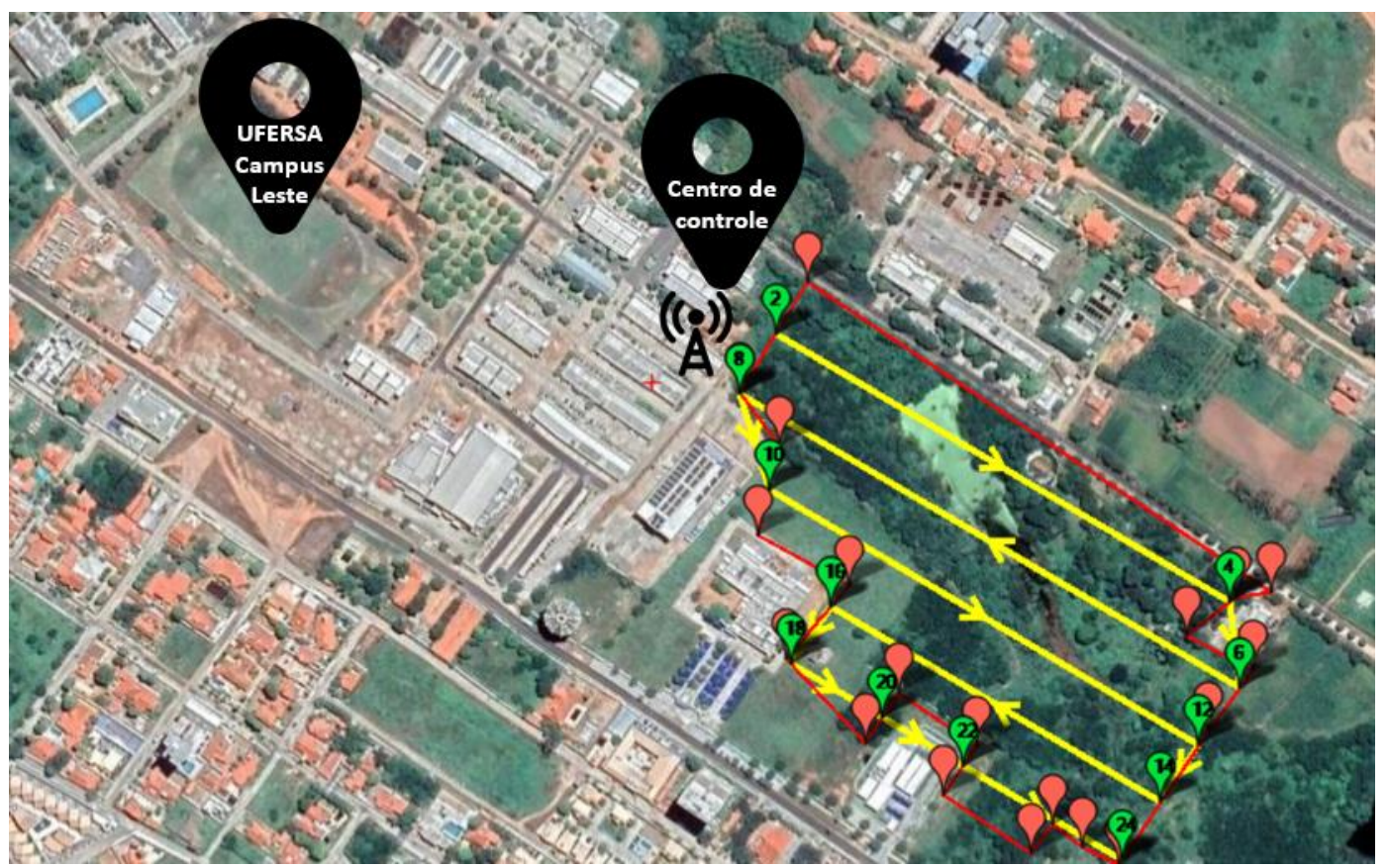

FIGURA 2. Mapa de satélite da área de simulação. 


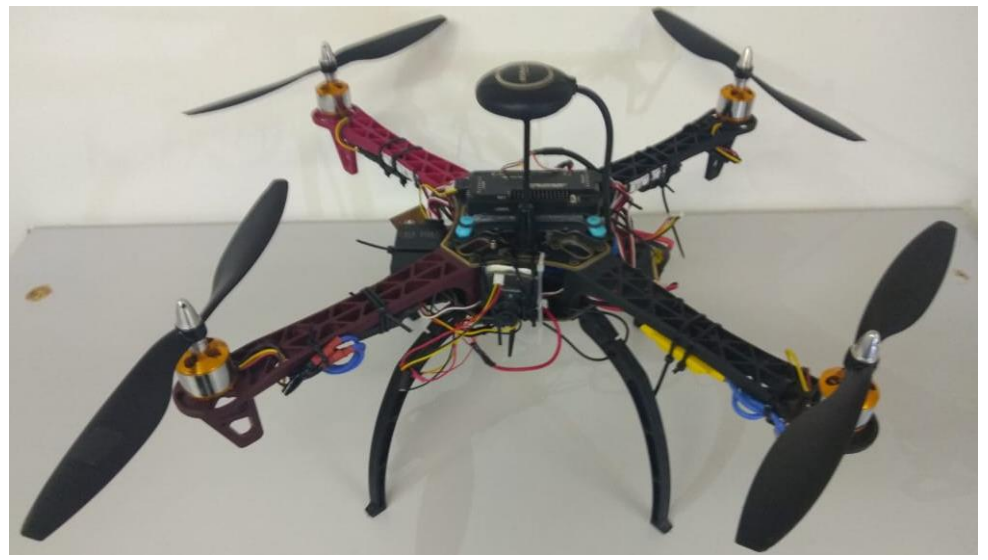

FIGURA 3. VANT modelo para simulações.

Com a área demarcada, foi simulado o percurso com capturas de imagens de acordo com a Figura 4, no qual, o VANT em questão teria o objetivo de mapear o local no qual está sendo plantado uma determinada cultura, para obter mapas de produtividade, infecção de pragas, plantas daninhas (plantas indesejáveis na produção), dentre outras. Com este tipo de processo, podem ser obtidos mapas como os das Figuras 5 e 6.

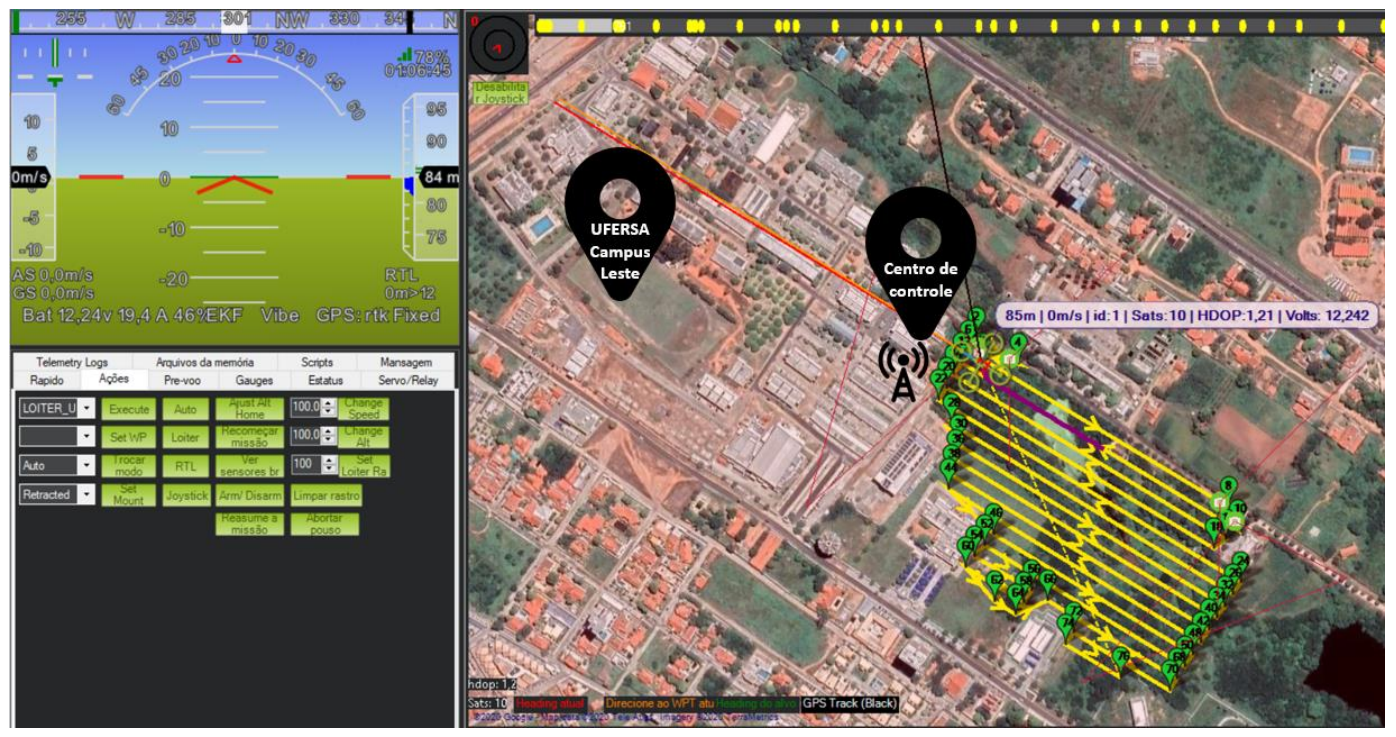

FIGURA 4. Mapa de satélite da simulação de voo.

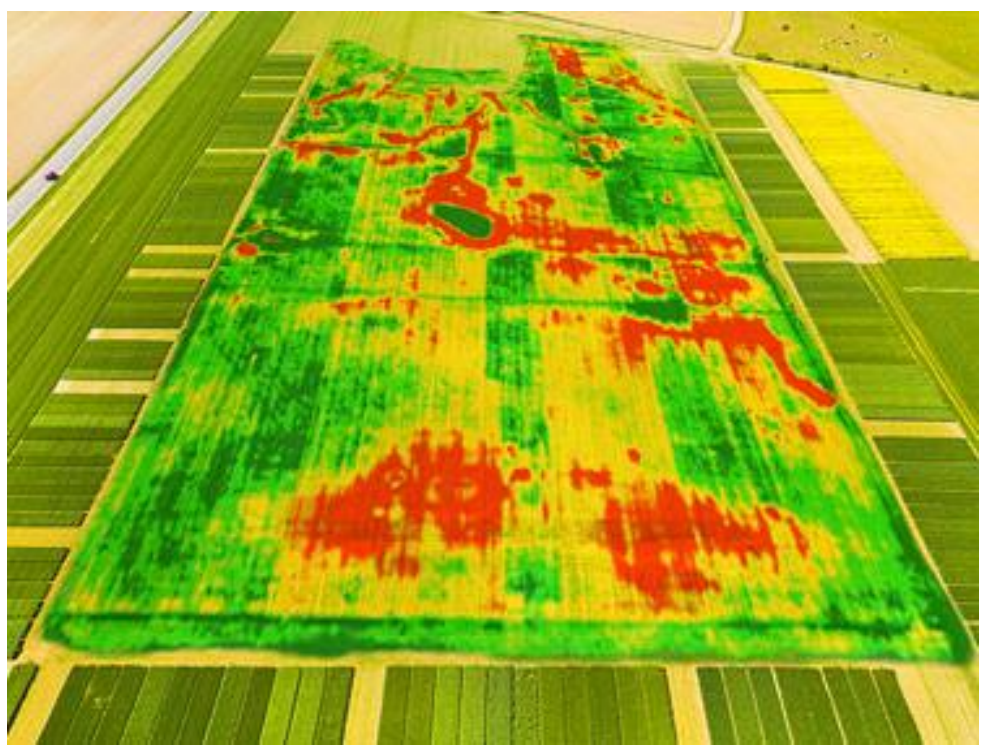

FIGURA 5. Mapa genérico. [6] 


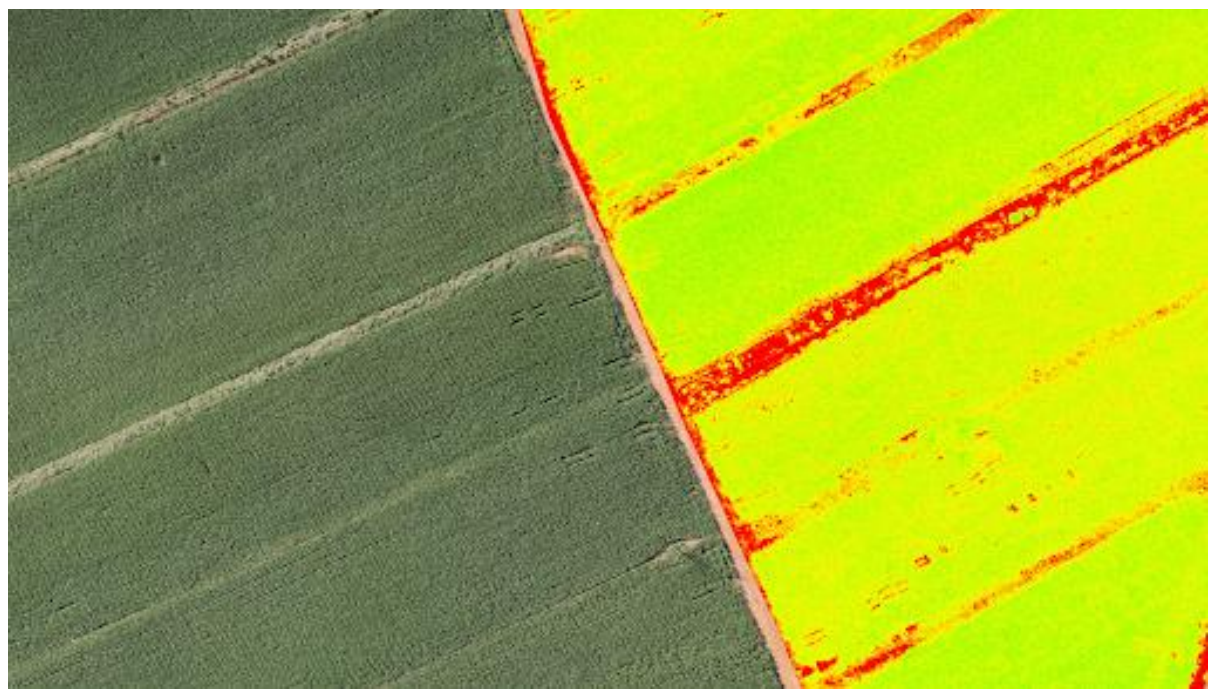

FIGURA 6. Mapa de produtividade. [1]

\section{RESUltAdOS}

\subsection{Perda de retorno}

Com as simulações realizadas no software Ansys HFSS 2019®, foi possível a identificação da frequência de ressonância da antena desenvolvida, comprovando os métodos numéricos e cálculos utilizados no Scilab®. A Figura 7 representa a perda de retorno da antena de microfita projetada, no qual, apresentou uma largura de banda de 94,1 MHz, característico de uma antena de banda larga, com uma perda de retorno de $-27,89 \mathrm{~dB}$ da frequência desejada, e desta maneira, confirmando a aplicabilidade da antena para a frequência de 2,45 GHz.

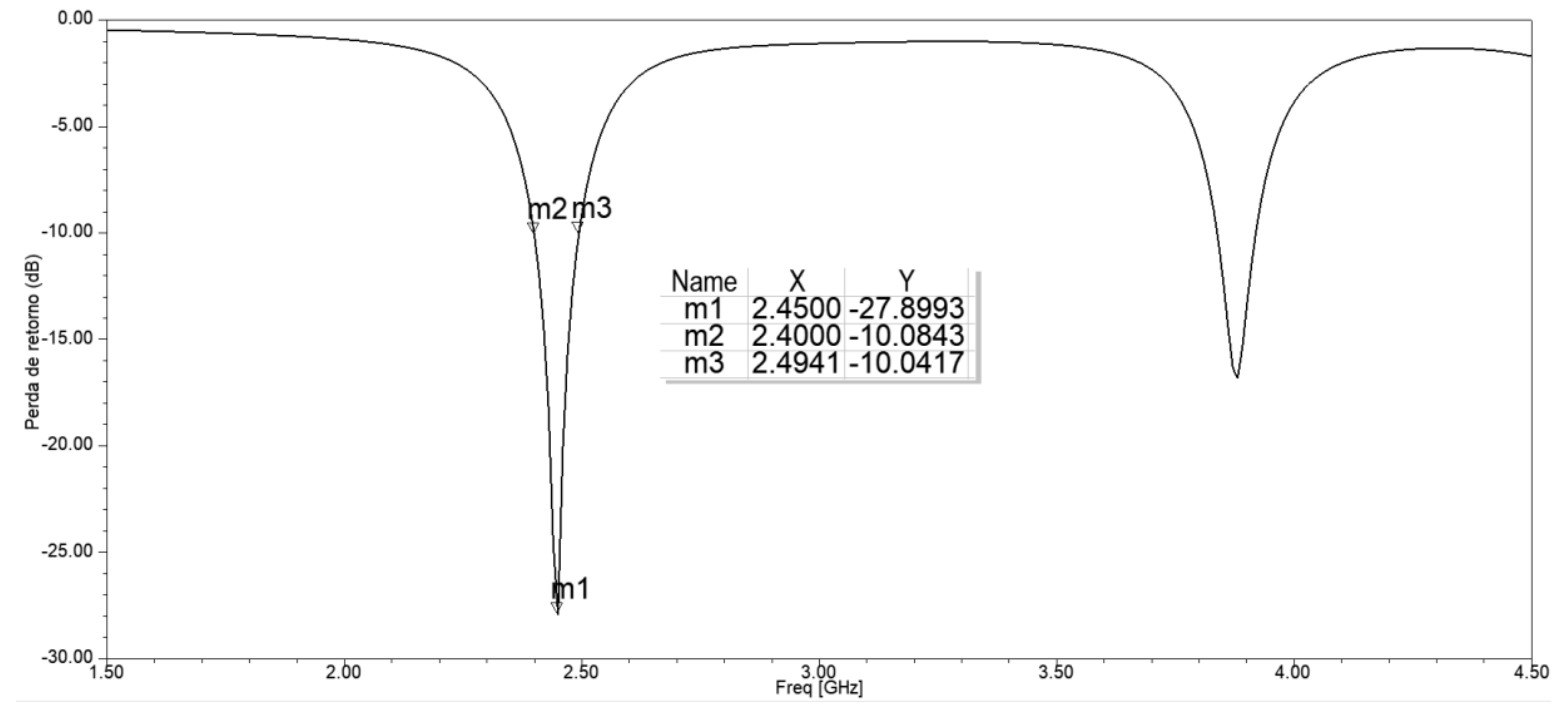

FIGURA 7. Perda de retorno da antena projetada.

\subsection{Diagramas de radiação $2 D$ e $3 D$}

Por conseguinte, através do mesmo software foi possível a identificar o comportamento da antena através dos diagramas de radiação $2 \mathrm{D}$ e $3 \mathrm{D}$ da antena de microfita. A Figura 8 representa cortes no diagrama tridimensional mostrado na Figura 9 para phi igual a $0^{\circ}$ e $90^{\circ}$, no qual, é uma representação tridimensional da correlação entre a potência fornecida e potência refletida da antena [7]. Portanto, o ganho máximo obtido pela antena foi de $0,68 \mathrm{~dB}$. O comportamento do campo visto na Figura 09 na antena de microfita pode ser observado de maneira mais conclusiva na Figura 10, demonstrando a característica diretiva da antena. 


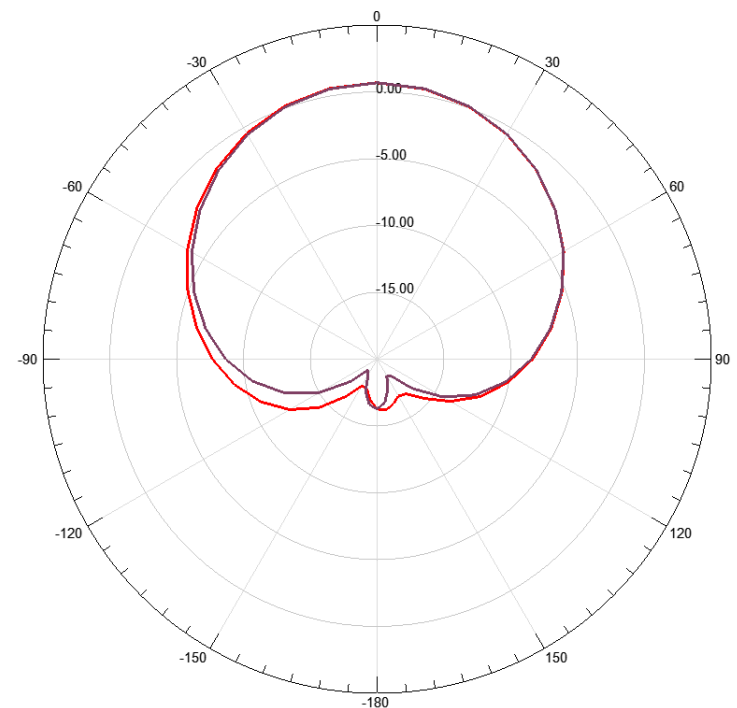

FIGURA 8. Diagrama de radiação 2D da antena projetada.

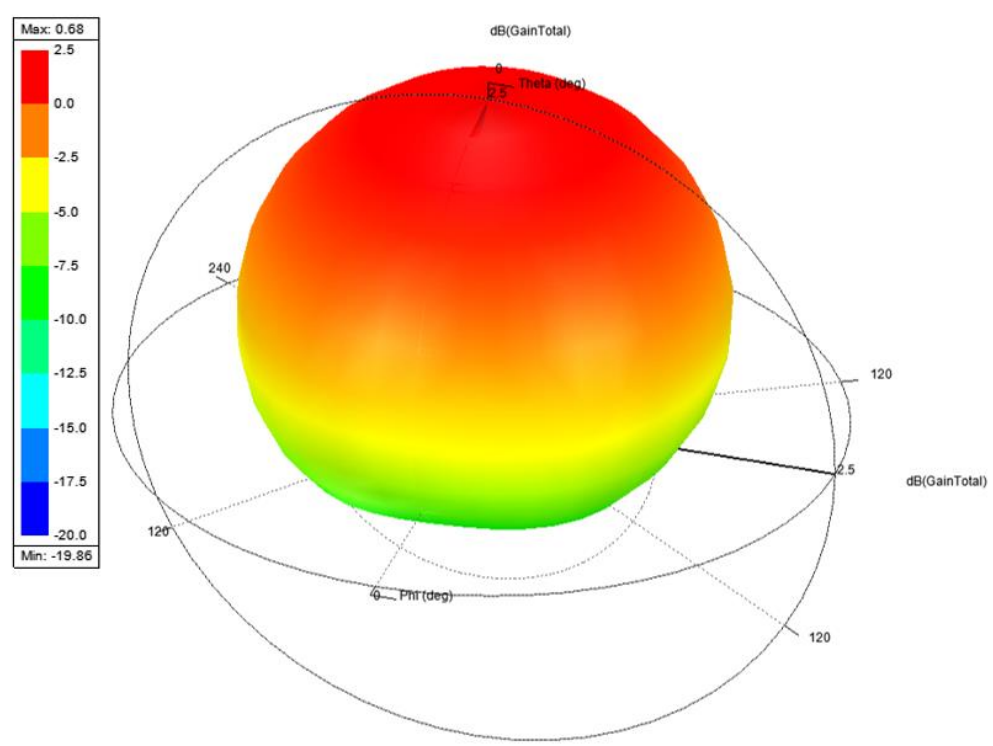

FIGURA 9. Diagrama de radiação 3D da antena projetada.

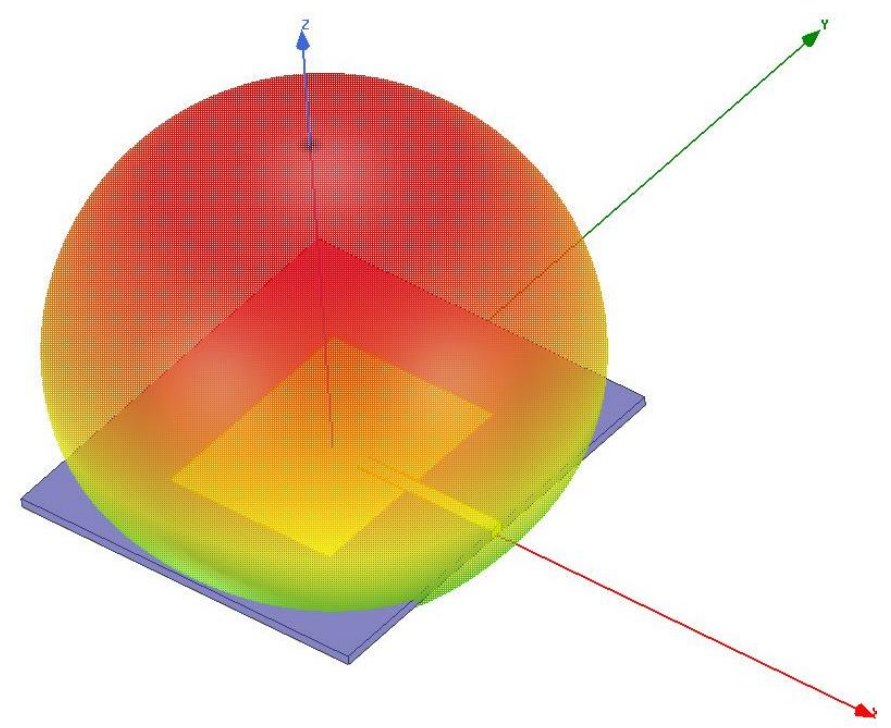

FIGURA 10. Diagrama de radiação 3D da antena projetada. 


\section{CONCLUSÃO}

Deve-se observar que a inserção dos VANT's em diversas áreas de estudo e mercados, se torna fundamental o desenvolvimento deste tipo de tecnologia, visto que, ainda possui um grande espaço para melhorias, por diversos problemas que hoje possui, como tempo reduzido de voo, devido a bateria; necessidade de um operador manualmente iniciar o mecanismo de monitoramento, para que após isto, o modo automático possa funcionar; além de, problemas de alcance de sinal, tema que levou a execução deste trabalho. Portanto, a antena projetada para este serviço se mostrou adequada, visto que alcançou os objetivos planejados, sendo assim, este tipo de configuração pode ser utilizado para situações na qual o dispositivo precise percorrer uma área maior em uma única direção. Desta maneira, a implementação de uma antena de microfita dentro da faixa de frequência se torna uma boa solução pelo baixo custo e perfil, além de permitir varrer com precisão as áreas especificadas e permitir um voo autônomo através do software Mission Planner ${ }^{\circledR}$

\section{AGRADECIMENTOS}

Os autores agradecem à CAPES, CNPq, UFERSA, GEPEAT e à equipe do Laboratório de Micro-ondas da UFERSA. O presente trabalho foi realizado com apoio da Coordenação de Aperfeiçoamento de Pessoal de Nível Superior - Brasil (CAPES) - Código de Financiamento 001.

\section{REFERÊNCIAS}

[1] Horus Aeronaves. (2019) “Agricultura de precisão com drones”. https://horusaeronaves. com/ebook/ebook-drones-na-agricultura-precisao-horus.pdf.

[2] Balanis, C. A. (2015) “Antenna Theory: Analysis and Design”. New Jersey (USA): John Wiley \& Sons, Inc.

[3] Silva, R. A. O. (2014) "Aplicação de Técnicas de Alargamento de Banda em Antenas de Microfita", M. Eng. tese, Instituto Militar de Engenharia, Rio de Janeiro, Brasil.

[4] J. P. P, PEREIRA. (2016) "Estruturas PBG periódicas e quase periódicas em Antenas de Microfita com aplicação em veículos aéreos não tripulados” D. Eng. Elétrica e computação, UFRN, Natal.

[5] Y. Huang e K. Boyle. (2008) “Antennas: From Theory to Practice”. New Delhi, India: John Wiley\& Sons, vol 1.

[6] GEODATA. (2020) “Como interpretar mapas de produtividade na agricultura de precisão". Disponível em: <https://www.geodataap.com.br/2019/10/14/mapas-produtividade-na-agricultura-precisao/>. Acesso em: 06 de Junho, 2020.

[7] Pozar, D. M. (2012) “Engenharia de micro-ondas”. 4 ed., Estados Unidos da América: John Wiley\& Sons. p. 756 . 